\title{
Effect of the iodine atom position on the phosphorescence of BODIPY derivatives: a combined computational and experimental study
}

\author{
Elena Bassan ${ }^{1}$ (D) Yasi Dai $^{1}$ (D) Daniele Fazzi ${ }^{1}$ D $\cdot$ Andrea Gualandi $^{1}$ (D) Pier Giorgio Cozzi ${ }^{1}$ (D) Fabrizia Negri ${ }^{1,2}$ (D) \\ Paola Ceroni ${ }^{1}$ D
}

Received: 4 October 2021 / Accepted: 1 December 2021 / Published online: 13 January 2022

( ) The Author(s), under exclusive licence to European Photochemistry Association, European Society for Photobiology 2021

\begin{abstract}
A new BODIPY derivative ( $o$-I-BDP) containing an iodine atom in the ortho position of the meso-linked phenyl group was prepared. Photophysical and electrochemical properties of the molecule were compared to previously reported iodo BODIPY derivatives, as well as to the non-iodinated analog. While in the case of derivatives featuring iodine substituents in the BODIPY core, efficient population of the triplet state is accompanied by a substantial positive shift of the reduction potential compared to pristine BODIPY, $o$-I-BDP displays phosphorescence and simultaneously maintains the electrochemical properties of unsubstituted BODIPYs. A theoretical investigation was settled to analyze results and rationalize the influence of iodine position on electronic and photophysical properties, with the purpose of preparing a fully organic phosphorescent BODIPY derivative. TD-DFT and spin-orbit coupling calculations shed light on the subtle effects played by the introduction of iodine atom in different positions of BODIPY.
\end{abstract}

\section{Graphical abstract}

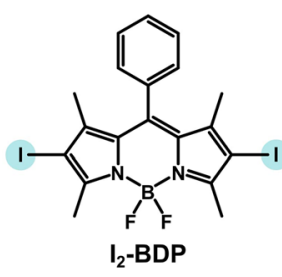

REDOX POTENTIALS $\boldsymbol{x}$ TRIPLET STATE

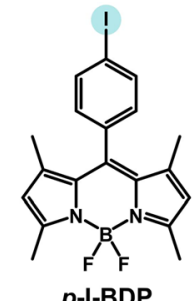

p-I-BDP

REDOX POTENTIALS

TRIPLET STATE $\otimes$
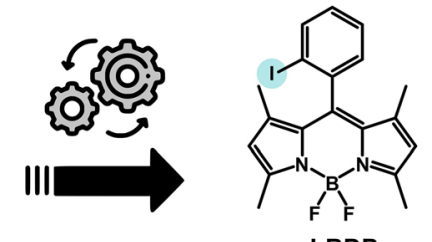

REDOX POTENTIALS

o-I-BDP
TRIPLET

STATE

Keywords Phosphorescence $\cdot$ Triplet state $\cdot$ DFT calculations $\cdot$ Spin-orbit coupling calculations $\cdot$ Singlet oxygen

Elena Bassan and Yasi Dai contributed equally to this work.

Dedicated to Angelo Albini on the occasion of his 75th birthday.

The corresponding authors are: Pier Giorgio Cozzi, (responsible for thesynthesis), Fabrizia Negri, (responsible for the computational part), Paola Ceroni, (responsible for the experimental optical and electrochemical properties)

Pier Giorgio Cozzi

piergiorgio.cozzi@unibo.it

Fabrizia Negri

fabrizia.negri@unibo.it

$\triangle$ Paola Ceroni

paola.ceroni@unibo.it

\section{Introduction}

BODIPYs (boron-dipyrromethenes) are a class of organoboron compounds that has long received much attention for their high molar absorption coefficients, high fluorescence

1 Department of Chemistry Giacomo Ciamician, University of Bologna, Bologna, Italy

2 INSTM, UdR Bologna, Via F. Selmi, 2, 40126 Bologna, Italy 
quantum yields and photostability. These properties have consequently led to a variety of applications, such as sensors for metal ions [1], fluorescent probes or labels targeted toward biological components [2].

The ease of their chemical modification has brought forth the development of derivatives that possess novel photophysical features. In particular, BODIPYs that efficiently populate their lowest triplet excited state [3] have been recently designed by three main strategies: (i) introduction of heavy atoms, either by halogenation or insertion of heavy metals [4]; (ii) coupling of BODIPY to electron donating chromophores in donor-acceptor dyads [5]; (iii) BODIPY dimers [6]. In the last two classes, the population of the $T_{1}$ excited state is usually mediated by a photoinduced charge-transfer process and requires the synthesis of quite complex systems, while halogenation is an easier approach and affects the rate of spin-forbidden radiative and non-radiative processes. For instance, when iodine atoms are introduced at the 2 and 6 positions (Fig. 1), intersystem crossing (ISC) becomes efficient and the lowest triplet state can be populated, as demonstrated by Nagano et al. in 2005 [7]. This non-radiative deactivation becomes faster thanks to the internal heavy atom effect, as commonly observed for metal complexes containing heavy metal ion, e.g., $\mathrm{Ru}$ (II) or Ir(III) complexes [8]. Halogenation of the BODIPY core has therefore been extensively investigated for the development of drugs in photodynamic therapy [9], as it enables

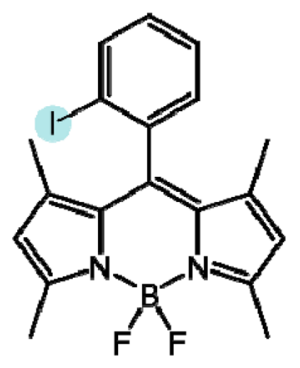

o-I-BDP

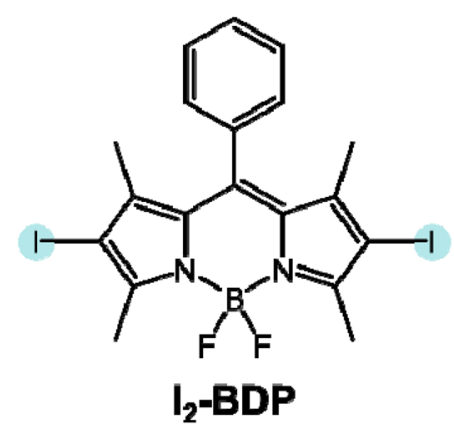

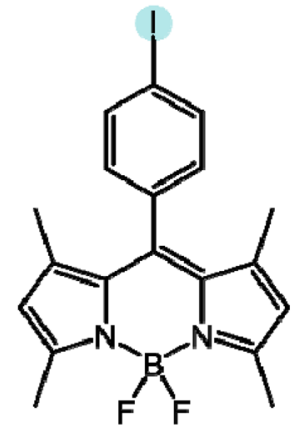

p-I-BDP

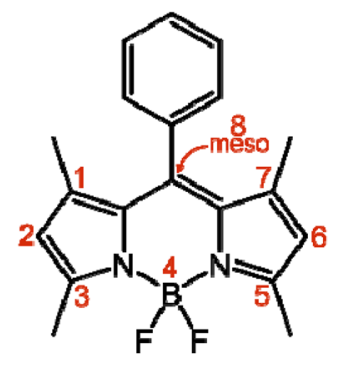

BDP
Fig. 1 Structure of the studied compounds and IUPAC numeration the production of cytotoxic singlet oxygen without the use of heavy metals. More recently, these derivatives have been used as photosensitizers in photocatalytic reactions [10], which has required taking into consideration the effect of halogenation on their electrochemical redox potentials as well.

This work focuses on a series of iodinated BODIPY derivatives (Fig. 1) and the rationalization of the effect that the position of the iodine atom has on the photophysical and electrochemical properties of such compounds. $\mathrm{I}_{2}-\mathrm{BDP}$ is characterized by the presence of two iodine atoms directly linked to the chromophoric unit, while $p$-I-BDP possesses one iodine atom in the para position of the meso-linked phenyl group. The new BODIPY derivative, $o$-I-BDP, was designed by placing one iodine atom in the ortho position of the phenyl group so that the halogen is placed close to the BODIPY core, but is not directly attached to it. The effect of this substitution was studied photophysically, electrochemically and computationally.

\section{Experimental and computational details}

\subsection{Synthesis of compounds}

2-iodobenzaldehyde [11], $p$-I-BDP [12] and $\mathrm{I}_{2}-\mathrm{BDP}$ [13] were prepared according to literature procedures.

Synthesis of $o$-I-BDP. Dipyrromethane was synthesized according to a modified literature procedure [14]. Deionized water $(17 \mathrm{~mL})$ was degassed in a Schlenk tube and 2-iodobenzaldehyde ( $0.42 \mathrm{mmol}, 97 \mathrm{mg}, 1$ eq.) was subsequently added. Distilled 2,4-dimethylpyrrole $(10.5 \mathrm{mmol}$, $1.00 \mathrm{~g}, 25$ eq.) was introduced, and the mixture was shielded from light and vigorously stirred at $80{ }^{\circ} \mathrm{C}$ for $24 \mathrm{~h}$. After cooling at room temperature, AcOEt $(20 \mathrm{~mL})$ was added and the organic phase was separated. Aqueous phase was extracted with AcOEt $(3 \times 20 \mathrm{~mL})$, and the collected organic phases were evaporated under reduced pressure to obtain a brown oil. The residue was filtrated on a silica plug (cyHex:DCM:AcOEt, 7:2:1), and the solvent was removed under reduced pressure to obtain dipyrromethane that was employed in the next step without further purification. Dipyrromethane was dissolved in anhydrous DCM $(25 \mathrm{~mL})$ and 2,3-dichloro-5,6-dicyano-1,4-benzoquinone (DDQ) ( $0.5 \mathrm{mmol}, 114 \mathrm{mg}, 1.2 \mathrm{eq}$.) was added. After stirring at r.t. for $12 \mathrm{~h}$, anhydrous $\mathrm{Et}_{3} \mathrm{~N}$ (25.2 mmol, $2.55 \mathrm{~g}, 60$ eq.) was added, followed, after $30 \mathrm{~min}$, by the addition of $\mathrm{BF}_{3} \cdot \mathrm{Et}_{2} \mathrm{O}$ ( $16.8 \mathrm{mmol}, 2.38 \mathrm{~g}, 40 \mathrm{eq}$.). The solution was stirred for $1 \mathrm{~h}$ and then filtered through a pad of Celite washing with DCM. Deionized water $(20 \mathrm{~mL})$ was added to the filtrate and the organic phase was separated. Aqueous phase was extracted with DCM $(3 \times 20 \mathrm{~mL})$, and the collected organic phases were evaporated under reduced pressure. $o$-I-BDP 
was purified by flash chromatography (cyHex:AcOEt, 4:1) to obtain an orange solid (37\% yield). ${ }^{1} \mathrm{H}$ NMR: $\left(\mathrm{CDCl}_{3}\right.$, $400 \mathrm{MHz}) \delta 1.38(\mathrm{~s}, 6 \mathrm{H}), 2.55(\mathrm{~s}, 6 \mathrm{H}), 5.98(\mathrm{~s}, 2 \mathrm{H}), 7.94$ $(\mathrm{d}, 1 \mathrm{H}, \mathrm{J}=8.0 \mathrm{~Hz}), 7.48(\mathrm{t}, 1 \mathrm{H}, \mathrm{J}=7.6 \mathrm{~Hz}), 7.29(\mathrm{~d}, 1 \mathrm{H}$, $\mathrm{J}=7.5 \mathrm{~Hz}), 7.15(\mathrm{t}, 1 \mathrm{H}, \mathrm{J}=7.7 \mathrm{~Hz}) ;{ }^{13} \mathrm{C}$ NMR $\left(\mathrm{CDCl}_{3}\right.$, $100 \mathrm{MHz}) \delta 156.0,142.4,139.9,139.6,130.4,129.0,128.9$, 121.2, 98.2, 14.7, 14.0; ${ }^{19} \mathrm{~F}$ NMR $\left(\mathrm{CDCl}_{3}, 376.5 \mathrm{MHz}\right) \delta$ 145.93 (ddd, 1F, J = 32.9, 66.4, 109.4 Hz), 146.66 (ddd, 1F, $\mathrm{J}=31.6,64.4,109.4 \mathrm{~Hz})$.

\subsection{Computational details}

Ground-state equilibrium structures of each molecule shown in Fig. 1 were determined with density functional theory (DFT) calculations. Two functionals were considered in this case, the B3LYP and the M06-2X. The 6-31G* basis set was used for all atoms except iodine for which the LANL2DZ basis set and pseudopotential [15] were employed. Solvent effects were included by means of the polarizable continuum model (PCM) [16]. According to the experimental measurements, $\mathrm{MeCN}(\varepsilon=35.688)$ was considered as solvent. The corresponding calculations are labeled as PCM-B3LYP/631G*/LANL2DZ and PCM-M06-2X/6-31G*/LANL2DZ. The nature of the stationary points determined by geometry optimization were verified by vibrational frequency calculations carried out at the same level of theory. All the reported geometries correspond to minima.

Excited state wavefunctions and excitation energies were calculated with time-dependent (TD)-DFT, using the M06-2X functional and the 6-311G* basis set, while iodine atoms were described as indicated above. In addition, selected TD-DFT calculations were carried out with the $6-311+\mathrm{G}^{*}$ and def2-TZVP basis sets. Ten triplet and ten singlet excited states were included in the calculations. Due to the constrained cyanine nature of BODIPY and the insufficient electron correlation at TD-DFT level, excitation energies are systematically overestimated by ca. $0.4-0.5 \mathrm{eV}$ [17-19] at this level of theory. Nevertheless, it has been shown in several benchmark works that the M06-2X functional is suitable to describe excitation energies in BODIPY derivatives and the variations induced by side groups, modifications of the skeleton, stiffening or extension of the conjugated path [19-22]. Excited state calculations are indicated as PCM-TD-M06-2X/6-311G*/LANL2DZ. Excitation energies in solution were initially determined with the use of the standard linear response (LR). Solute-solvent polarization effects in excited states were additionally determined with the state specific (SS) corrected linear response (cLR) $[23,24]$ approach. Only the fast solvent component was equilibrated (hereafter indicated as NEQ) in calculations of excited state energies used to model absorption spectra, while a fully equilibrated solvent was assumed (hereafter labeled EQ) for excited state energies employed in the discussion of photo-deactivation processes. Accordingly, the solvent correction included in excited state calculations is indicated by the label LR, cLR(NEQ) or cLR(EQ). All calculations were performed with the Gaussian 16 package [25].

For spin-orbit coupling (SOC) calculations, we considered both PCM-B3LYP (Figure S1 and Table S1) and PCMM06-2X (Figure S2 and Table S2) geometries. The SOC integrals were calculated with the spin-orbit mean-field (SOMF) method, with one-center approximation applied to the exchange term, $(\operatorname{SOMF}(1 \mathrm{X}))[27,28]$. Relativistic corrections were included with the zeroth order regular approximation (ZORA) [29] using the basis sets specifically designed for these all-electrons calculations: SARC-ZORATZVP for iodine and ZORA-def2-TZVP for the remaining atoms. The calculations were carried out with ORCA 5.0.1 package [26] with the Tamm-Dancoff approximation (TDA) [30].

\section{Results and discussion}

\subsection{Synthesis}

The preparation of $o$-I-BDP was carried out with a slight modification of a synthetic procedure previously reported by one of us [14]. The synthesis takes advantage of the standard approach employed for BODIPY dyes. A dipyrromethane, obtained in a convenient green "on water" procedure starting from $o$-iodobenzaldehyde, is oxidized with DDQ, and, without purification, the derivative is treated with $\mathrm{BF}_{3}$ ether complex in the presence of an excess of base $\left(\mathrm{Et}_{3} \mathrm{~N}\right)$. Compared to other procedures published in the literature, the "on water" procedure reduces the excess of 2,5-dimethylpyrrole and avoids Lewis or Brønsted acids, limiting decomposition or polypyrrolic products. The final BODIPY is conveniently purified by chromatography, in moderate isolated yields.

\subsection{Photophysical characterization}

The photophysical properties of $o$-I-BDP were studied in air-equilibrated acetonitrile solution and compared to those of $\mathrm{I}_{2}-\mathrm{BDP}, p-\mathrm{I}-\mathrm{BDP}$ and BDP so as to rationalize the effects brought about by iodination at different sites of the BODIPY core. The absorption spectrum of $o$-I-BDP (Fig. 2B, solid line) presents a narrow band that corresponds to the $S_{0} \rightarrow S_{1}$ electronic transition $\left(\lambda_{\max }=503 \mathrm{~nm}, \varepsilon=63,600 \mathrm{M}^{-1} \mathrm{~cm}^{-1}\right)$. This band is blue-shifted compared to that of $\mathrm{I}_{2}-\mathrm{BDP}$ and slightly red-shifted compared to that of $p$-I-BDP and BDP [31] (Table 1). The emission spectrum recorded at room temperature (Fig. 2B, dashed line) peaks at $521 \mathrm{~nm}$ and is attributable to $o$-I-BDP's fluorescence, given its small Stokes shift and its lifetime of $5.0 \mathrm{~ns}$. The same trend 


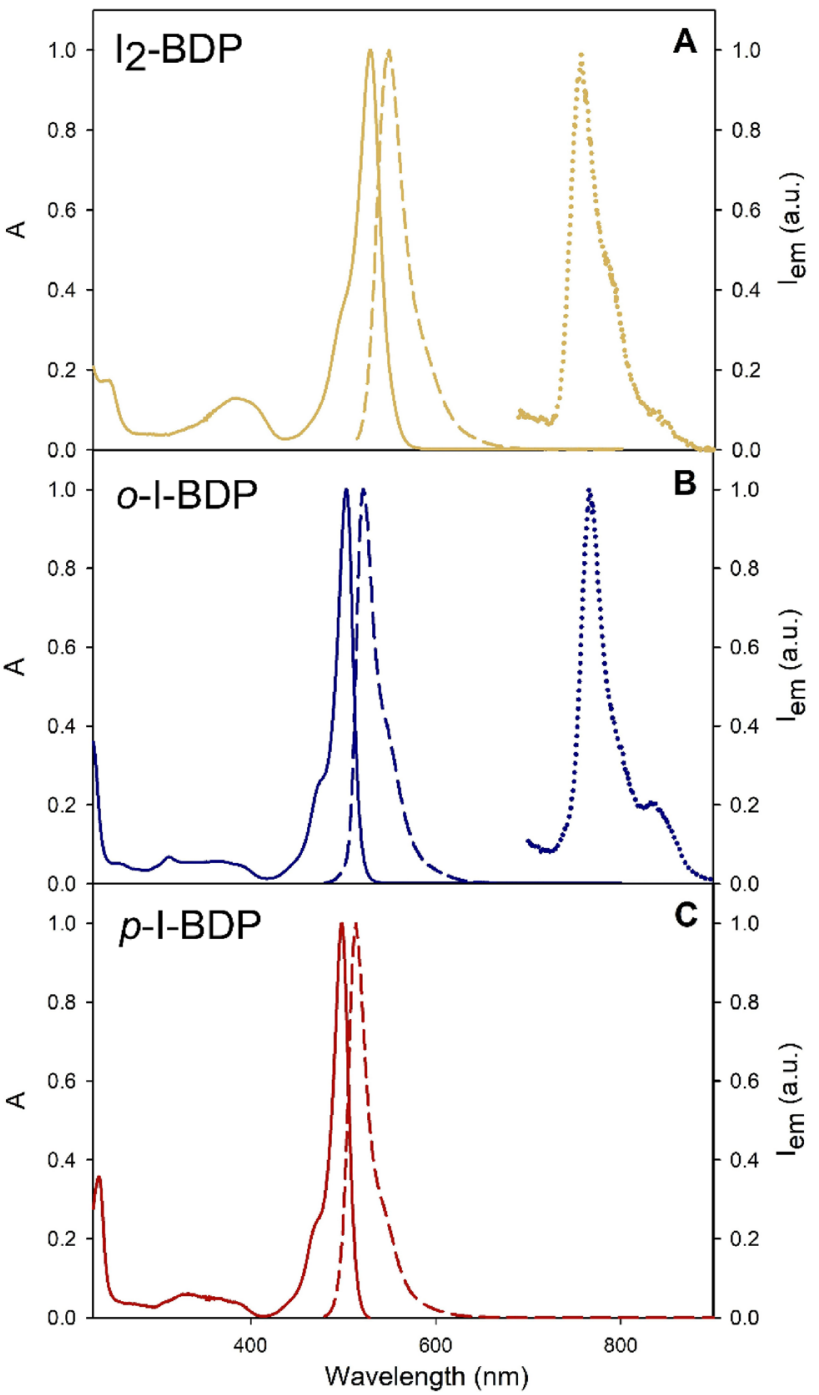

Fig. 2 Absorption (solid line), fluorescence (dashed line), and phosphorescence (dotted line) spectra of $\mathrm{I}_{2}-\mathrm{BDP}(\mathbf{A})$, o-I-BDP (B) and p-I-BDP (C)

observed for the absorption maxima is maintained for the fluorescence maxima as well (Table 1).
Interestingly, $o$-I-BDP presents a new emission band $\left(\lambda_{\max }=765 \mathrm{~nm}\right)$ at $77 \mathrm{~K}$ in an $\mathrm{EtOH} / \mathrm{MeOH}(1: 1 \mathrm{v} / \mathrm{v})$ glassy matrix with a lifetime of $520 \mu \mathrm{s}$ (Fig. 2B, dotted line). This band is assigned to $o$-I-BDP's phosphorescence due to its larger Stokes shift, its absence in aerated solutions at room temperature and its decay lifetime. $\mathrm{I}_{2}-\mathrm{BDP}$ also presents a similar band in the same spectral region, whereas $p$-I-BDP does not (Fig. 2A, C, and Table 1). These results are ascribable to the different positions occupied by iodine atoms, which strongly influence the efficiency of ISC between the lowest singlet $\left(S_{1}\right)$ and triplet states, as well as the efficiency of phosphorescence. In fact, given the distance of the iodine atom from the chromophoric core, the ability of $p$-I-BDP to populate its lowest triplet state and thus display phosphorescence is strongly reduced and its photophysical properties resemble those of non-iodinated BDP [31, 32]. Conversely, ISC is favored in $\mathrm{I}_{2}-\mathrm{BDP}$ since the two iodine atoms are directly attached to the BODIPY skeleton. In the case of the $o$-I-BDP derivative, the presence of one iodine atom in the vicinity of the BODIPY core allows it to populate the lowest triplet excited state, even though it is not directly appended to it.

To understand the extent of this deactivation pathway, fluorescence quantum yields $\left(\Phi_{\text {FLUO }}\right)$ and singlet oxygen quantum yields $\left(\Phi_{\Delta}\right)$ were measured for the three compounds (Table 1) and compared to those of BDP. Fluorescence quantum yield is expected to decrease upon increasing the rate of ISC and, consequently, $\Phi_{\Delta}$ is expected to increase because singlet oxygen is generated by energy transfer from the lowest triplet excited state $\left(T_{1}\right)$ of the BODIPY (Fig. 3).

In accordance with literature data [33], $\mathrm{I}_{2}-\mathrm{BDP}$ is characterized by the lowest value of $\Phi_{\text {FLUO }}$ and the highest value of $\Phi_{\Delta}$ because the two iodine atoms are positioned in such a way that they are highly effective at inducing ISC and obtaining an efficient $\mathrm{T}_{1}$ population. In addition to the efficient ISC process, a more stable $S_{1} / S_{0}$ conical intersection could also contribute to the reduced $\Phi_{\text {FLUO }}$ of $\mathrm{I}_{2}-\mathrm{BDP}$ [34]. $o$-I-BDP's $\Phi_{\mathrm{FLUO}}(56 \%)$ is similar to that of $p$-I-BDP but, remarkably, $\Phi_{\Delta}$ is $35 \%$ for $o$-I-BDP and
Table 1 Photophysical properties of studied compounds in air-equilibrated $\mathrm{MeCN}$ solution

\begin{tabular}{llllllll}
\hline Molecule & $\lambda_{\max \text { ABS }}(\mathrm{nm})$ & $\lambda_{\max \text { FLUO }}(\mathrm{nm})$ & $\tau_{\text {FLUO }}(\mathrm{ns})$ & $\Phi_{\text {FLUO }}$ & $\lambda_{\max \text { PHOS }}(\mathrm{nm})^{\mathrm{a}}$ & $\tau_{\text {PHOS }}(\mathrm{ms})^{\mathrm{a}}$ & $\Phi_{\Delta}$ \\
\hline$o$-I-BDP & 503 & 521 & 5.0 & $56 \%$ & 765 & 0.52 & $35 \%$ \\
$p$-I-BDP & 498 & 514 & 3.3 & $45 \%$ & $\rho^{\mathrm{b}}$ & $/$ & $\rho^{\mathrm{b}}$ \\
$\mathrm{I}_{2}-\mathrm{BDP}$ & 530 & 548 & $/ \mathrm{c}$ & $1.8 \%$ & 757 & 1.20 & $70 \%$ \\
BDP [31-33] & 497 & 507 & 3.18 & $48 \%$ & n.r. & n.r. & $1 \%^{\mathrm{d}}$ \\
\hline
\end{tabular}

n.r. not reported

${ }^{a}$ At $77 \mathrm{~K}$ in $\mathrm{MeOH} / \mathrm{EtOH}(1: 1 \mathrm{v} / \mathrm{v})$

${ }^{\mathrm{b}}$ Not detected

${ }^{c}$ Lifetime not determined due to low value of $\Phi_{\mathrm{FLUO}}$

${ }^{\mathrm{d}}$ In DCM 


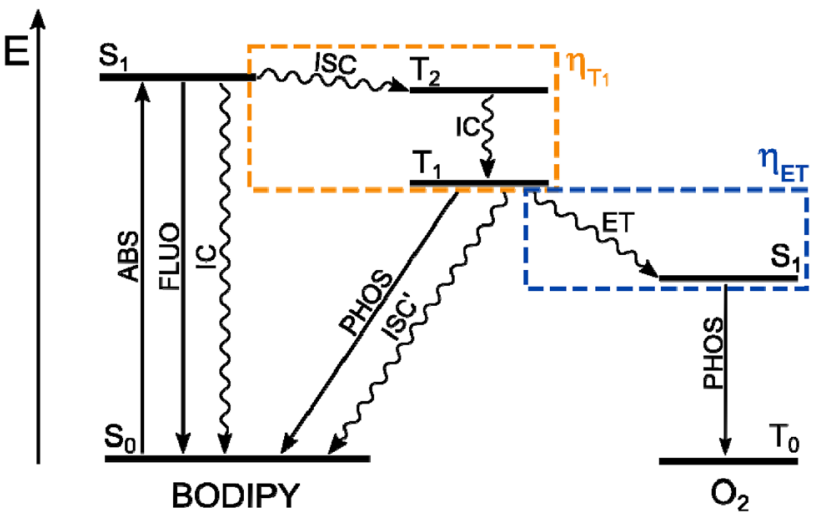

Fig. 3 Jablonski diagram illustrating the main energy levels and deactivation pathways for BODIPY derivatives in the presence of molecular oxygen; $\eta_{T_{1}}$ is the efficiency of $T_{1}$ population and $\eta_{\mathrm{ET}}$ is the efficiency of the bimolecular energy transfer process between BODIPY and ground-state molecular oxygen. The role of the $T_{2}$ state will be discussed in the computational section

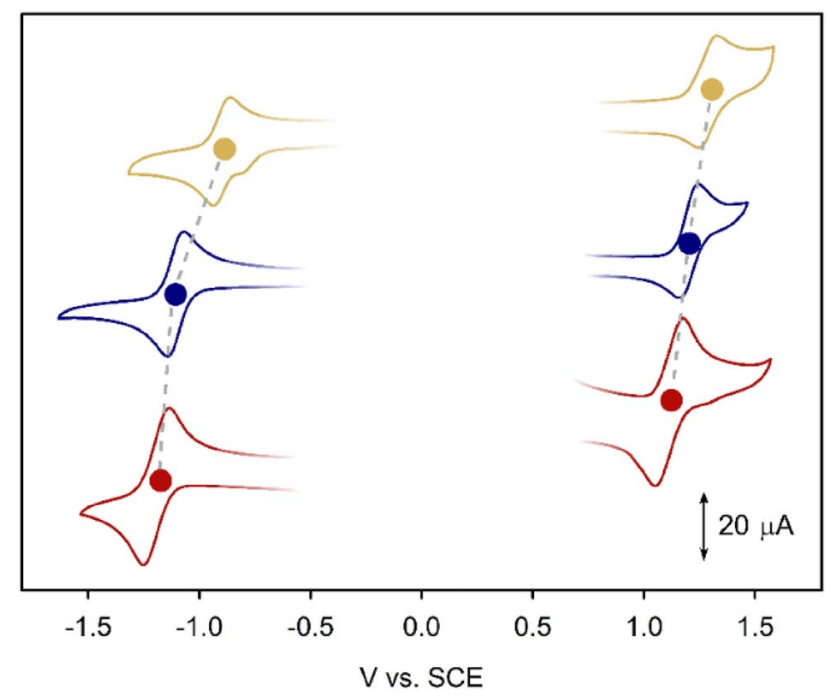

Fig. 4 Cyclic voltammetry of $\mathrm{I}_{2}-\mathrm{BDP}$ (yellow line), o-I-BDP (blue line) and $\mathrm{p}-\mathrm{I}-\mathrm{BDP}$ (red line) $(0.5 \mathrm{mM})$ in degassed $\mathrm{MeCN}$ solution containing tetraethylammonium hexafluorophosphate $0.05 \mathrm{M}$, as the supporting electrolyte and ferrocene as the internal standard. Working electrode: glassy carbon; counter electrode: Pt wire; quasi-reference electrode: Ag wire; scan rate $=0.3 \mathrm{~V} / \mathrm{s}$. Ferrocene's peaks have been removed for clarity

not detectable for $p$-I-BDP. These results indicate that the location of the iodine atom is crucial for the population of the triplet state: $o$-I-BDP exhibits a quite efficient $T_{1}$ population, although not as efficient as in $\mathrm{I}_{2}-\mathrm{BDP}$. On the contrary, $p$-I-BDP is unable to undergo efficient ISC, similarly to what is reported for BDP [33]. Lastly, the higher $\Phi_{\text {FLUO }}$ of $o-\mathrm{I}-\mathrm{BDP}$ compared to $p$-I-BDP can be imputed to the increased steric hindrance of its meso substituent. In fact, a substituent in the ortho position decreases its rotational freedom and, in turn, its non-radiative deactivation.

\subsection{Electrochemical characterization}

The ground-state electrochemical potentials of the three BODIPY derivatives were studied by means of cyclic voltammetry (Fig. 4). $o$-I-BDP shows chemically and electrochemically reversible electron transfer processes, both in the anodic (+1.20 V vs. SCE) and cathodic region $(-1.10 \mathrm{~V}$ vs . SCE) (Fig. 4, blue line).

Both these redox potentials are negatively shifted compared to those of $\mathrm{I}_{2}-\mathrm{BDP}$, and are comparable to those measured for $p$-I-BDP and BDP [13], as presented in Fig. 4. Indeed, the presence of halogen atoms directly connected to the BODIPY core reduces its electron density, while derivatizations present on the meso-linked phenyl group have little influence on the electrochemical properties thanks to its orthogonality with respect to the chromophoric unit. Therefore, iodination in the ortho position of the phenyl group allows to maintain the redox potentials typical of fluorescent BODIPYs meanwhile allowing the population of their lowest triplet excited state.

\subsection{Modeling photophysical properties of BDP and iodinated derivatives}

To assist the interpretation of experimental results and rationalize the influence of iodine position, a computational investigation on the electronic structure and photophysical properties was carried out on BODIPY derivatives shown in Fig. 1.

\subsubsection{Ground-state structures and frontier molecular orbitals}

The computed equilibrium structures and bond lengths of the four BODIPY derivatives are collected in Figures S1, S2. We note that while PCM-B3LYP/6-31G*/LANL2DZ predicts an almost planar BODIPY core (the only exception is $\mathrm{I}_{2}-\mathrm{BDP}$ ) and an almost orthogonal phenyl moiety for all molecules (see Figure S1 and Table S1), PCM-M062X/6-31G*/LANL2DZ geometries display deviations from orthogonality for the phenyl substituent, except for $o$-I-BDP, and are characterized by a more markedly out of plane tilted $\mathrm{BF}_{2}$ group, as indicated by the dihedral angles in Table $\mathrm{S} 2$. In Fig. 5 and Figure S4, we see that the HOMO $(\mathrm{H})$ and LUMO (L), calculated at both levels of theory, are localized on the BODIPY core. Focusing on the set of PCM-B3LYP molecular orbitals (MOs, Fig. 5), we note that the shape of the $\mathrm{H}$ is the same for all molecules except for $\mathrm{I}_{2}-\mathrm{BDP}$. In the latter case significant electron density is computed also on the two iodine atoms. The electron density of $\mathrm{L}$ is 


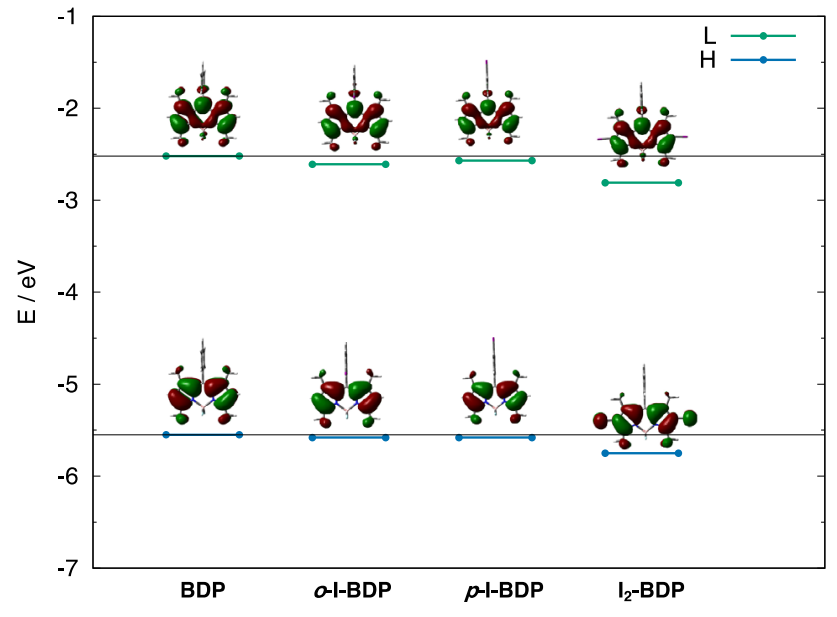

Fig. 5 Shapes and energies of $\mathrm{H}$ and $\mathrm{L}$ of BDP and iodinated derivatives calculated at PCM-B3LYP/6-31G*/LANL2DZ level

almost the same across the four molecules but due to the electron withdrawing character of iodine and its substitution on the BODIPY core, the LUMO energy of $\mathrm{I}_{2}-\mathrm{BDP}$ is significantly lowered. The substituent effect can be qualitatively explained via orbital interaction between fragments, or more quantitatively rationalized via absolutely localized molecular orbitals as demonstrated in recent work [35]. Due to the deviation from orthogonality, the MOs computed at PCM-M06-2X/6-31G*/LANL2DZ geometries (Figure S4), display some minor density contribution to phenyl substituents, especially for $\mathrm{I}_{2}-\mathrm{BDP}$.

Accurate computational predictions of redox potentials require comparison of energies for both the starting molecule and its reduced/oxidized forms. However, we can qualitatively use Koopmans' theorem [36] to correlate the redox potentials of BODIPY derivatives with $\mathrm{H} / \mathrm{L}$ computed

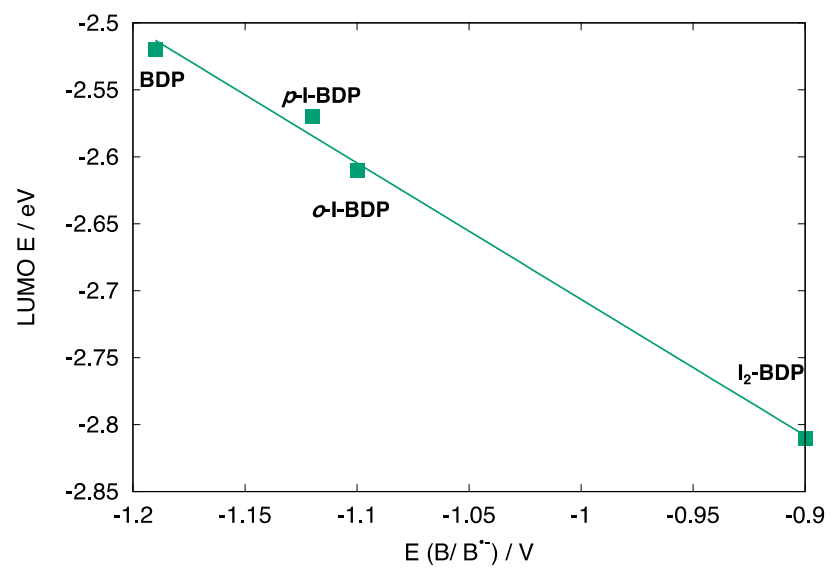

Fig. 6 Relationship between the $\mathrm{B} / \mathrm{B}^{--}$redox potentials (see Table 2) and L energies of BODIPY derivatives calculated at PCM-B3LYP/6$31 G^{*} /$ LANL2DZ level (see Table S3)
Table 2 Redox potentials of the studied compounds in $\mathrm{MeCN}$ in $\mathrm{V}$ vs. SCE

\begin{tabular}{lll}
\hline Molecule & $\mathrm{E}\left(\mathrm{B} / \mathrm{B}^{--}\right)(\mathrm{V})$ & $\mathrm{E}\left(\mathrm{B}^{\cdot+} / \mathrm{B}\right)(\mathrm{V})$ \\
\hline$o-\mathrm{I}-\mathrm{BDP}$ & -1.10 & +1.20 \\
$p$-I-BDP & -1.12 & +1.18 \\
$\mathrm{I}_{2}-\mathrm{BDP}$ & -0.90 & +1.29 \\
BDP [13] & -1.19 & +1.21 \\
\hline
\end{tabular}

energies. The result of such correlations is shown in Fig. 6 and S5, where a plot of the computed $\mathrm{L} / \mathrm{H}$ energies versus experimental redox potentials shows good linearity especially for $\mathrm{L}$ energies. The fitting of $\mathrm{H}$ energies versus $\mathrm{B}^{++} / \mathrm{B}$ redox potentials shows some deviations: BDP's oxidation potential is slightly higher than those of $o$-I-BDP and $p$-I-BDP. It should be noted however that these differences are small and are within experimental uncertainties. Interestingly, both markedly different redox potentials of $\mathrm{I}_{2}-\mathrm{BDP}$ are correctly captured by computations.

\subsubsection{Low-lying excited states}

To identify the deactivation pathways of the four BODIPY derivatives, we determined the most relevant excitation energies and excited state wavefunctions with PCM-TDM06-2X calculations, using the LANL2DZ basis set for iodine and three different basis sets for the remaining atoms: 6-311G*, 6-311+ $\mathrm{G}^{*}$ and def2-TZVP (including the standard LR description of solvent effects) at the PCMB3LYP/6-31G*/LANL2DZ optimized ground-state geometries (see Table S4). The corresponding molecular orbitals are depicted in Figure S6. As expected, the $S_{1}$ and $T_{1}$ states are dominated by the $\mathrm{H} \rightarrow \mathrm{L}$ excitation, localized on the BODIPY core. In addition to the lowest triplet state, in Table S4 we also report the excitation energy of the second lowest triplet $T_{2}$, which is computed to be very close to $S_{1}$ and is also dominated by an excitation localized on the BODIPY core, for all the systems investigated. Since the use of larger basis sets (see Table S4) leads to negligible differences in computed excitation energies, the following calculations were carried out with the $6-311 \mathrm{G}^{*}$ basis set.

The quality of predicted excited states can be assessed by comparing computed absorption spectra in solution with the experimental data. To this end, we determined the cLR(NEQ) corrected excitation energy of $S_{1}$ (see Table S5), the state responsible for the main absorption band of BODIPY derivatives. The comparison between computed and experimental spectra is shown in Fig. 7 where a shift of $0.45 \mathrm{eV}$ was applied to computed excitation energies to account for the systematic overestimation of $S_{1}$ excitation energy at TD-DFT level [17-19], as described in Sect. 2.2. 


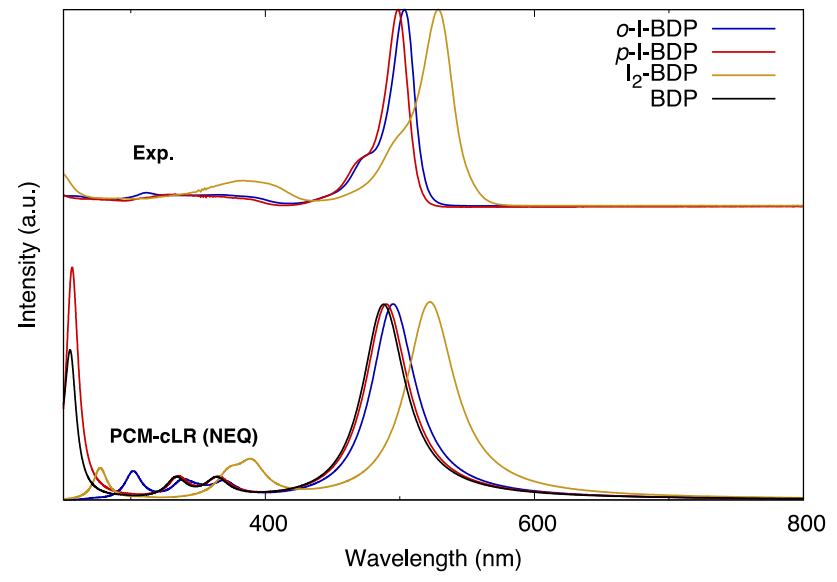

Fig. 7 Comparison between computed and observed absorption spectra of the four investigated BODIPY derivatives. PCM-TD-M062X/6-311G* level of theory including cLR(NEQ) solvent corrections. The computed excitation energies were shifted by $0.45 \mathrm{eV}$ in accordance with the known systematic overestimate at TD-DFT level [18]

Interestingly, the spectral changes induced by different iodination are very well reproduced by the calculations.

It is instructive to analyze in more detail the results of different computational protocols for solvent corrections. Solvatochromism in absorption spectra is determined by the different dipole moment of ground and excited states. Calculations show that the dipole moment (see Table S6) is systematically lower in $S_{1}$ compared to the ground state, for all the BODIPY derivatives investigated. This implies that an inverted solvatochromism [37] should be observed, accompanied by a blue shift when moving from gas-phase (calculations in vacuo) to MeCN. In contrast (see Table S5), $\mathrm{S}_{0} \rightarrow \mathrm{S}_{1}$ excitation energies computed in $\mathrm{MeCN}$ with the LR approach, decrease compared to gas-phase, while the expected excitation energy increase is predicted only by cLR(NEQ) calculations. Consider, for example the $S_{1}$ excitation energy computed at $2.97 \mathrm{eV}$ in vacuo for BDP, which unexpectedly decreases to $2.87 \mathrm{eV}$ in $\mathrm{MeCN}$ (LR) and correctly increases to $2.99 \mathrm{eV}$ in $\mathrm{MeCN}$ (cLR(NEQ)). As a result of the incorrect LR description, the correspondingly computed spectra are red-shifted, in Figure S8, compared to those obtained including the cLR(NEQ) solvent correction. The overshooting of positive solvatochromism by the LR approach has been documented in previous work [21, 38]. Here, we underscore that, for BODIPY derivatives characterized by inverted solvatochromism, the inclusion of solvent correction at LR level, incorrectly predicts an excitation energy decrease and only cLR provides a physically sound description of gas phase to solvent excitation energy changes.

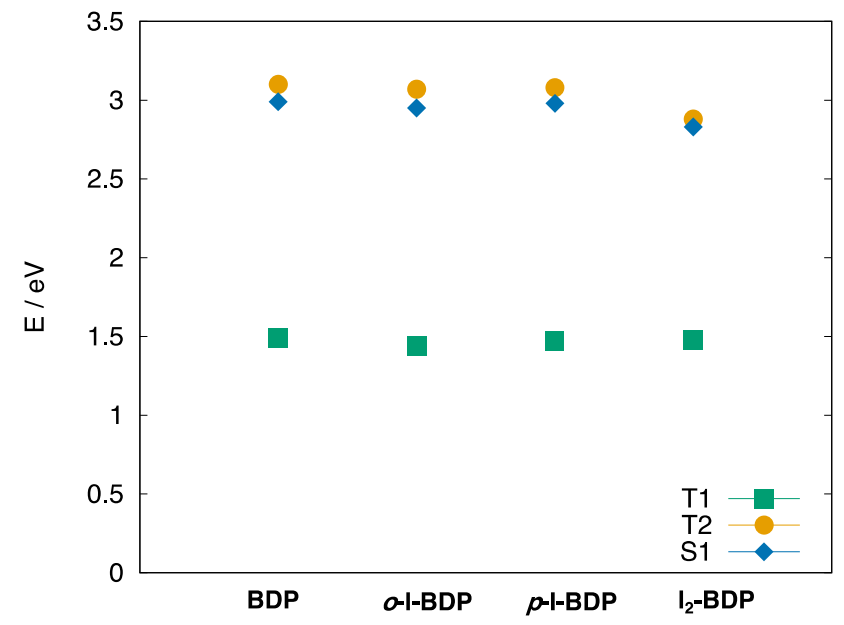

Fig. 8 Computed excitation energies (PCM-TD-M06-2X/6-311G*/ LANL2DZ level, including cLR(EQ) solvent correction) of low-lying singlet and triplet excited states of BDP and iodinated derivatives

\subsubsection{Predicted efficiency of ISC deactivation}

Experimental data (Table 1) show that only $o$-I-BDP and $\mathrm{I}_{2}-\mathrm{BDP}$ populate efficiently the lowest triplet state $T_{1}$ and exhibit relevant $\Phi_{\Delta}$ values. Furthermore, the efficiency is greater for $\mathrm{I}_{2}-\mathrm{BDP}$ with respect to $o$-I-BDP while population of $T_{1}$ is an inefficient process for $p$-I-BDP and BDP. According to the excited state calculations discussed in the previous section, two triplet states lie below $\left(T_{1}\right)$ or close $\left(T_{2}\right)$ to $S_{1}$ and should be considered to rationalize the experimental results. Because the ISC process is relatively slow [39] (of the order of $10^{7}-10^{8} \mathrm{~s}^{-1}$ ) compared to typical timescales of solvent reorganization [40] (picosecond, subpicosecond), we can safely assume that solvent equilibration occurs. Thus, solvent-equilibrated excited state energies (cLR(EQ)) (see Fig. 8) are considered in the following discussion. As discussed above, the $S_{1}$ state (blue diamond, Fig. 8) is found between $T_{1}$ and $T_{2}$ states, and quite close to the latter. Therefore, the $S_{1} \rightarrow T_{2}$ ISC cannot be ruled out. Indeed, in recent investigations on different BODIPY derivatives [41, 42] it has been proposed that the $S_{1} \rightarrow T_{2}$ deactivation path can be a relevant ISC channel.

For radiationless transitions slower than vibrational relaxation [43], and for relatively large energy gaps $\Delta E_{S-T}$ between $S_{1} / T_{n}$ states, the ISC process can be discussed in terms of a simplified relation (Eq. 1), where the rate constant $\left(k_{I S C}\right)$ is proportional to the SOC matrix element $\left\langle T_{n}\left|\widehat{H_{S O}}\right| S_{1}\right\rangle$ and inversely proportional to the square of the energy gap $\Delta E_{S-T}$ between $\mathrm{S}_{1} / \mathrm{T}_{\mathrm{n}}$ states [41, 43-45]: 
Table 3 Computed SOC magnitudes between $S_{1}$ and $T_{1} / T_{2}$ states of $\mathrm{BDP}$ and iodinated derivatives

\begin{tabular}{lll}
\hline \multirow{2}{*}{ Molecule } & \multicolumn{2}{l}{ SOC @ geometry PCM-B3LYP } \\
\cline { 2 - 3 } & $\begin{array}{l}S_{1} / T_{1}\left[\mathrm{~cm}^{-1}\right]^{\mathrm{a}} \\
\left(\Delta \mathrm{E}_{\mathrm{S}-\mathrm{T}}[\mathrm{eV}]\right)^{\mathrm{b}}\end{array}$ & $\begin{array}{l}S_{1} / T_{2}\left[\mathrm{~cm}^{-1}\right]^{\mathrm{a}} \\
\left(\Delta \mathrm{E}_{\mathrm{S}-\mathrm{T}}[\mathrm{eV}]\right)^{\mathrm{b}}\end{array}$ \\
\hline$o-\mathrm{I}-\mathrm{BDP}$ & $1.09(1.51)$ & $0.14(-0.11)$ \\
$p-\mathrm{I}-\mathrm{BDP}$ & $0.00(1.51)$ & $0.00(-0.11)$ \\
I - BDP & $2.64(1.35)$ & $11.70(-0.05)$ \\
BDP & $0.00(1.50)$ & $0.00(-0.11)$ \\
\hline
\end{tabular}

a SOC values calculated with the $\operatorname{SOMF}(1 \mathrm{X})$ approach including relativistic corrections with ZORA, from TDA-M06-2X/ZORA-def2TZVP/SARC-ZORA-TZVP calculations at PCM-B3LYP/6-31G* (BDP) and PCM-B3LYP/6-31G*/LANL2DZ (iodinated BDP) optimized geometries

${ }^{\mathrm{b}} \Delta \mathrm{E}_{\mathrm{S}-\mathrm{T}}$ from PCM-TD-M06-2X/6-311G* and PCM-TD-M06-2X/6$311 \mathrm{G} * / \mathrm{LANL} 2 \mathrm{DZ}$ levels, including cLR(EQ) solvent correction

$k_{I S C} \propto \frac{\left|\left\langle T_{n}\left|\widehat{H_{S O}}\right| S_{1}\right\rangle\right|^{2}}{\left(\Delta E_{S-T}\right)^{2}}$.

The SOCs between $T_{1}, T_{2}$ and $S_{1}$, computed at the optimized PCM-B3LYP (PCM-M06-2X) geometries are collected in Table 3 (Table S7 and S8). In the same table we also report the relevant $\Delta E_{S-T}$ values taken from cLR(EQ) calculations (Fig. 8, Table S5). Although the two sets of geometries display different degrees of orthogonality between the phenyl substituent and the BODIPY core and a different $\mathrm{BF}_{2}$ out of plane tilt, it is interesting to note that both consistently suggest a similar decay pattern. The SOCs computed for the two geometries of $o$-I-BDP are very similar since this is the only case in which the BODIPY derivative displays an orthogonal geometry also at PCM-M06-2X level. Concerning the magnitude of computed SOCs, we note that these are negligible for BDP, both at PCM-B3LYP and PCM-M06-2X geometries. For $p$-I-BDP the computed SOCs are also negligible for both sets of geometries, although slightly larger at the less symmetric PCM-M06-2X structure. A markedly different trend is documented for the remaining two iodinated derivatives. $o$-I-BDP displays, for both geometries, a relevant SOC between $T_{1}$ and $S_{1}$ and a non-negligible value between $S_{1}$ and $T_{2}$. It is well known that the presence of a second triplet state in the vicinity of the excited singlet greatly enhances the rate of the intersystem crossing [46]. Since $\Delta E_{S-T}$ is considerably smaller for the $T_{2}$ state, we cannot rule out a contribution from the $S_{1} \rightarrow T_{2}$ ISC to the efficient triplet formation. Finally, very large SOCs are computed for $\mathrm{I}_{2}-\mathrm{BDP}$ in perfect agreement with the remarkable effect experimentally documented for iodine atoms directly linked to the BODIPY core. While both SOCs between $T_{1} / S_{1}$ and $T_{2} / S_{1}$ are significant, we note that the less symmetric PCM-M06-2X structure leads to larger couplings, suggesting that vibronically induced effects might also be relevant. At the same time the $\left\langle T_{2}\left|\widehat{H_{S O}}\right| S_{1}\right\rangle$ couplings are much larger than those computed for $o-\mathrm{I}-\mathrm{BDP}$. This, coupled to a reduction of the $S_{1} / T_{2}$ energy difference in $\mathrm{I}_{2}-\mathrm{BDP}$, points to an even more relevant role of the $S_{1} \rightarrow T_{2}$ ISC for this system.

Thus, based on the above considerations, we expect negligible triplet generation for BDP and $p$-I-BDP. The most efficient triplet generation is predicted for $\mathrm{I}_{2}-\mathrm{BDP}$ while an intermediate efficiency is expected for $o$-I-BDP. A possible additional deactivation pathway via $S_{1} \rightarrow T_{2}$ ISC is predicted for both, with an increased efficiency for $\mathrm{I}_{2}-\mathrm{BDP}$. These conclusions are in agreement with experimental data.

\section{Conclusions}

The purpose of this work was the synthesis of fully organic phosphorescent BODIPY derivatives. Given the improvements in the photophysical properties recently displayed by this class of molecules, the effort was aimed at the synthesis of BODIPYs that did not contain heavy metals, but relied on iodine atoms for the efficient population of their triplet states. Monoiodination in the ortho position of meso phenyl-BODIPY proved to be an effective way to gain access to the phosphorescent triplet state while maintaining fluorescent BODIPYs electrochemical properties. The molecules' photophysical and electrochemical properties are therefore decidedly influenced by the positions that iodine atoms occupy around the BODIPY core, and their rationalization was possible on the basis of the combined experimental and computational study.

Quantum chemical calculations were shown to support the remarkable changes in redox potentials of $\mathrm{I}_{2}-\mathrm{BDP}$ and the relative shifts in absorption spectra along the series of BODIPY derivatives investigated. From a methodological point of view, it was shown that only the cLR approach correctly describes solvatochromic effects in BODIPY derivatives, when moving from gas-phase to solvent. Finally, the computed low-lying excited states and SOCs were shown to be fully consistent with the inefficient triplet generation of BDP and $p$-I-BDP. The superior efficiency of $\mathrm{I}_{2}-\mathrm{BDP}$ compared to $o$-I-BDP was demonstrated by large SOCs for the two available ISC pathways: $S_{1} \rightarrow T_{2}$ and $S_{1} \rightarrow T_{1}$. While functionalization of iodine atoms at different positions in BDP does not affect substantially the nature of low-lying excited states, it modulates their relative energy. The tiny changes in wavefunctions induced by the presence and position of iodine atoms are nicely captured by the calculated SOCs which provide a consistent interpretation of the experimental data. 
These results are useful for the design of other BODIPY derivatives exhibiting highly efficient population of the lowest triplet state with potential applications in photocatalysis and photodynamic therapy.

Supplementary Information The online version contains supplementary material available at https://doi.org/10.1007/s43630-021-00152-5.

Funding The National PRIN 2017 projects (ID: 20174SYJAF, SURSUMCAT and ID: 20172M3K5N, CHIRALAB) are acknowledged for financial support for this research. Fondazione CarisBo is acknowledged for the funding of the project "Tecnologie avanzate per il controllo e lo sviluppo di molecole innovative per la salute" (ID: \#18668). The University of Bologna is gratefully acknowledged.

\section{Declarations}

Conflict of interest The authors declare that they have no conflict of interest.

\section{References}

1. Niu, L. Y., Li, H., Feng, L., Guan, Y. S., Chen, Y. Z., Duan, C. F., Wu, L. Z., Guan, Y. F., Tung, C. H., \& Yang, Q. Z. (2013). BODIPY-based fluorometric sensor array for the highly sensitive identification of heavy-metal ions. Analytica Chimica Acta, 775, 93-99.

2. Kowada, T., \& Kikuchi, K. (2015). BODIPY-based probes for the fluorescence imaging of biomolecules in living cells. Chemical Society Reviews, 14, 4953-4972.

3. Bassan, E., Gualandi, A., Cozzi, P. G., \& Ceroni, P. (2021). Design of BODIPY dyes as triplet photosensitizers: Electronic properties tailored for solar energy conversion, photoredox catalysis and photodynamic therapy. Chemical Science, 12, 6607-6628.

4. Zhao, J., Xu, K., Yang, W., Wang, Z., \& Zhong, F. (2015). The triplet excited state of Bodipy: Formation, modulation and application. Chemical Society Reviews, 44, 8904-8939.

5. Filatov, M. A. (2019). Heavy-atom-free BODIPY photosensitizers with intersystem crossing mediated by intramolecular photoinduced electron transfer. Organic \& Biomolecular Chemistry, 18, $10-27$.

6. Ventura, B., Marconi, G., Bröring, M., Krüger, R., \& Flamigni, L. (2009). Bis(BF2)-2,2'-bidipyrrins, a class of BODIPY dyes with new spectroscopic and photophysical properties. New Journal of Chemistry, 33, 428-438.

7. Yogo, T., Urano, Y., Ishitsuka, Y., Maniwa, F., \& Nagano, T. (2005). Highly efficient and photostable photosensitizer based on BODIPY chromophore. Journal of the American Chemical Society, 127, 12162-12163.

8. Balzani, V., Ceroni, P., \& Juris, A. (2014). Photochemistry and photophysics. Concepts, research, application. Weinheim: Wiley.

9. Kamkaew, A., Lim, S. H., Lee, H. B., Kiew, L. V., Chung, L. Y., \& Burgess, K. (2013). BODIPY dyes in photodynamic therapy. Chemical Society Reviews, 42, 77-88.

10. Magagnano, G., Gualandi, A., Marchini, M., Mengozzi, L., Ceroni, P., \& Cozzi, P. G. (2017). Photocatalytic ATRA reaction promoted by iodo-Bodipy and sodium ascorbate. Chemical Communications, 53, 1591-1594.

11. Boelke, A., Lork, E., \& Nachtsheim, B. J. (2018). N-heterocyclestabilized iodanes: From structure to reactivity. Chemistry A European Journal, 24, 18653-18657.
12. Xu, Y., Chang, D., Feng, S., Zhang, C., \& Jiang, J. X. (2016). BODIPY-containing porous organic polymers for gas adsorption. New Journal of Chemistry, 40, 9415-9423.

13. Zhang, C., Zhao, J., Wu, S., Wang, Z., Wu, W., Ma, J., Guo, S., \& Huang, L. (2013). Intramolecular RET enhanced visible lightabsorbing bodipy organic triplet photosensitizers and application in photooxidation and triplet-triplet annihilation upconversion. Journal of the American Chemical Society, 135, 10566-10578.

14. Zoli, L., \& Cozzi, P. G. (2009). Electrophilic activation of aldehydes 'on water': A facile route to dipyrromethanes. Chemsuschem, 2, 218-220.

15. Wadt, W. R., \& Hay, P. J. (1985). Ab initio effective core potentials for molecular calculations. Potentials for main group elements $\mathrm{Na}$ to Bi. The Journal of Chemical Physics, 82, 284-298.

16. Tomasi, J., Mennucci, B., \& Cammi, R. (2005). Quantum mechanical continuum solvation models. Chemical Reviews, 105, 2999-3093.

17. Postils, V., Ruipérez, F., \& Casanova, D. (2021). Mild open-shell character of BODIPY and its impact on singlet and triplet excitation energies. Journal of Chemical Theory and Computation, 17, $5825-5838$.

18. Zinna, F., Bruhn, T., Guido, C. A., Ahrens, J., Bröring, M., Di Bari, L., \& Pescitelli, G. (2016). Circularly polarized luminescence from axially chiral BODIPY DYEmers: An experimental and computational study. Chemistry A European Journal, 22, 16089-16098.

19. Chibani, S., Laurent, A. D., Le Guennic, B., \& Jacquemin, D. (2014). Improving the accuracy of excited-state simulations of BODIPY and Aza-BODIPY dyes with a joint SOS-CIS(D) and TD-DFT approach. Journal of Chemical Theory and Computation, 10, 4574-4582.

20. Chibani, S., Le Guennic, B., Charaf-Eddin, A., Laurent, A. D., \& Jacquemin, D. (2013). Revisiting the optical signatures of BODIPY with ab initio tools. Chemical Science, 4, 1950-1963.

21. Charaf-Eddin, A., Le Guennic, B., \& Jacquemin, D. (2014). Excited-states of BODIPY-cyanines: Ultimate TD-DFT challenges? RSC Advances, 4, 49449-49456.

22. Laine, M., Barbosa, N. A., Wieczorek, R., Melnikov, M. Y., \& Filarowski, A. (2016). Calculations of BODIPY dyes in the ground and excited states using the M06-2X and PBE0 functionals. Journal of Molecular Modeling, 22, 260.

23. Mennucci, B. (2015). Modeling absorption and fluorescence solvatochromism with QM/Classical approaches. International Journal of Quantum Chemistry, 115, 1202-1208.

24. Caricato, M., Mennucci, B., Tomasi, J., Ingrosso, F., Cammi, R., Corni, S., \& Scalmani, G. (2006). Formation and relaxation of excited states in solution: a new time dependent polarizable continuum model based on time dependent density functional theory. The Journal of Chemical Physics, 124, 124520.

25. Frisch, M. J., Trucks, G. W., Schlegel. H. B., Scuseria, G. E., Robb, M. A., Cheeseman, J. R., Scalmani, G., Barone, V., Petersson, G. A., Nakatsuji, H. X., Li, X., Caricato, M., Marenich, A. V., Bloino, J., Janesko, B. G., Gomperts, R., Mennucci, B., Hratchian, H. P., Ortiz, J. V., ...Fox, D. J. (2016) Gaussian, Inc., Wallingford CT

26. Neese, F., Wennmohs, F., Becker, U., \& Riplinger, C. (2020). The ORCA quantum chemistry program package. The Journal of Chemical Physics, 152, 224108.

27. De Souza, B., Farias, G., Neese, F., \& Izsák, R. (2019). Predicting phosphorescence rates of light organic molecules using time-dependent density functional theory and the path integral approach to dynamics. Journal of Chemical Theory and Computation, 15, 1896-1904.

28. Neese, F. (2005). Efficient and accurate approximations to the molecular spin-orbit coupling operator and their use in molecular 
g-tensor calculations. The Journal of Chemical Physics, 122, 034107.

29. van Lenthe, E., Snijders, J. G., \& Baerends, E. J. (1996). The zero-order regular approximation for relativistic effects: The effect of spin-orbit coupling in closed shell molecules. The Journal of Chemical Physics, 105, 6505-6516.

30. Hirata, S., \& Head-Gordon, M. (1999). Time-dependent density functional theory within the Tamm-Dancoff approximation. Chemical Physics Letters, 314, 291-299.

31. Gabe, Y., Urano, Y., Kikuchi, K., Kojima, H., \& Nagano, T. (2004). Highly sensitive fluorescence probes for nitric oxide based on boron Dipyrromethene chromophore-Rational design of potentially useful bioimaging fluorescence probe. Journal of the American Chemical Society, 126, 3357-3367.

32. Duvva, N., Sudhakar, K., Badgurjar, D., Chitta, R., \& Giribabu, L. (2015). Spacer controlled photo-induced intramolecular electron transfer in a series of phenothiazine-boron dipyrromethene donoracceptor dyads. Journal of Photochemistry and Photobiology A: Chemistry, 312, 8-19.

33. Li, W., Li, L., Xiao, H., Qi, R., Huang, Y., Xie, Z., Jing, X., \& Zhang, H. (2013). Iodo-BODIPY: A visible-light-driven, highly efficient and photostable metal-free organic photocatalyst. RSC Advances, 3, 13417-13421.

34. Lin, Z., Kohn, A. W., \& Van Voorhis, T. (2020). Toward prediction of nonradiative decay pathways in organic compounds II: Two internal conversion channels in BODIPYs. Journal of Physical Chemistry C, 124, 3925-3938.

35. Mao, Y., Head-Gordon, M., \& Shao, Y. (2018). Unraveling substituent effects on frontier orbitals of conjugated molecules using an absolutely localized molecular orbital based analysis. Chemical Science, 9, 8598-8607.

36. Koopmans, T. (1934). Über die Zuordnung von Wellenfunktionen und Eigenwerten zu den Einzelnen Elektronen Eines Atoms. Physica, 1, 104-113.

37. Sunahara, H., Urano, Y., Kojima, H., \& Nagano, T. (2007). Design and synthesis of a library of BODIPY-based environmental polarity sensors utilizing photoinduced electron-transfer-controlled fluorescence ON/OFF switching. Journal of the American Chemical Society, 129, 5597-5604.

38. Chibani, S., Charaf-Eddin, A., Le Guennic, B., \& Jacquemin, D. (2013). Boranil and related NBO dyes: Insights from theory. Journal of Chemical Theory and Computation, 9, 3127-3135.

39. Buck, J. T., Boudreau, A. M., DeCarmine, A., Wilson, R. W., Hampsey, J., \& Mani, T. (2019). Spin-allowed transitions control the formation of triplet excited states in orthogonal donor-acceptor dyads. Chem, 5, 138-155.

40. Park, S., Kim, J., Moran, A. M., \& Scherer, N. F. (2011). Solvent structural relaxation dynamics in dipolar solvation studied by resonant pump polarizability response spectroscopy. Physical Chemistry Chemical Physics: PCCP, 13, 214-223.

41. Dong, Y., Kumar, P., Maity, P., Kurganskii, I., Li, S., Elmali, A., Zhao, J., Escudero, D., Wu, H., Karatay, A., Mohammed, O. F., \& Fedin, M. (2021). Twisted BODIPY derivative: Intersystem crossing, electron spin polarization and application as a novel photodynamic therapy reagent. Physical Chemistry Chemical Physics: PCCP, 23, 8641-8652.

42. Valiev, R. R., Cherepanov, V. N., Baryshnikov, G. V., \& Sundholm, D. (2018). First-principles method for calculating the rate constants of internal-conversion and intersystem-crossing transitions. Physical Chemistry Chemical Physics: PCCP, 20, 6121-6133.

43. Plotnikov, V. G. (1979). Regularities of the processes of radiationless conversion in polyatomic molecules. International Journal of Quantum Chemistry, 16, 527-541.

44. Chen, Y.-L., Li, S.-W., Chi, Y., Cheng, Y.-M., Pu, S.-C., Yeh, Y.-S., \& Chou, P.-T. (2005). Switching luminescent properties in osmium-based $\beta$-Diketonate complexes. ChemPhysChem, 6 , 2012-2017.

45. Zhang, J., Mukamel, S., \& Jiang, J. (2020). Aggregation-induced intersystem crossing: Rational design for phosphorescence manipulation. The Journal of Physical Chemistry B, 124, 2238-2244.

46. Bixon, M., \& Jortner, J. (1968). Intramolecular radiationless transitions. The Journal of Chemical Physics, 48, 715-726. 